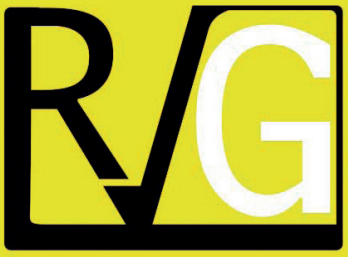

Julio - Septiembre, 2021

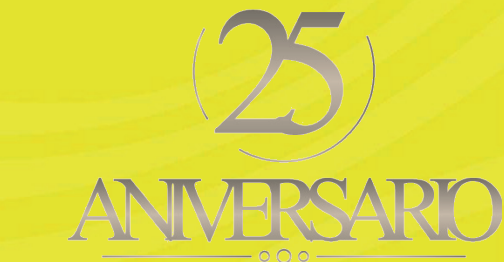

ANMERSARO
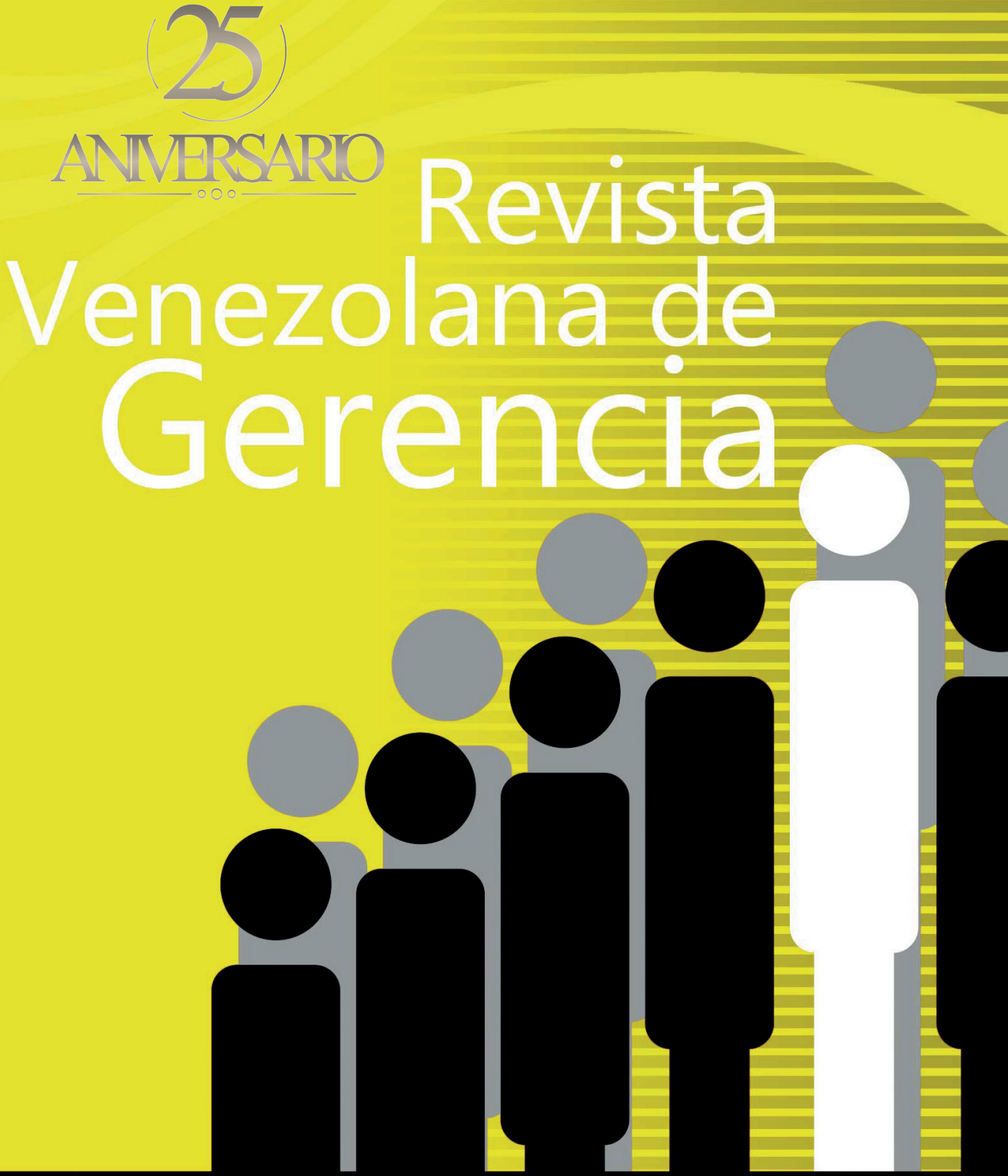

UNIVERSIDAD DEL ZULIA (LUZ)

Facultad de Ciencias Económicas y Sociales Centro de Estudios de la Empresa

ISSN 1315-99

Esta obra está bajo una licencia de Creative Comm Reconocimiento-NoComercial-Compartirlgual 3.0 Unpo http://creativecommons.org/licenses/by-nc-sa/3.0/deed.es 
COMO CITAR: Pérez Peralta, C. M., Chirinos Araque, Y., Ramírez García, A. G., y Barbera Alvarado, N. (2021). Política pública para el desarrollo local inclusivo desde la prospectiva estratégica en Colombia. Revista Venezolana de Gerencia (RVG), 26(95), 492-513. https://doi. org/10.52080/rvgluz.27.95.4
Universidad del Zulia (LUZ)

Revista Venezolana de Gerencia (RVG)

Año 26 No. 95 Julio-Septiembre 2021, 492-513

ISSN 1315-9984 / e-ISSN 2477-9423

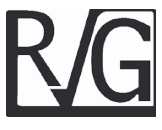

\title{
Política pública para el desarrollo local inclusivo desde la prospectiva estratégica en Colombia
}

\author{
Pérez Peralta, Claudia Milena* \\ Chirinos Araque, Yamarú del Valle** \\ Ramírez García, Adán Guillermo*** \\ Barbera Alvarado, Nataliya****
}

\section{Resumen}

El artículo tiene como objetivo determinar elementos para la formulación de una política pública de desarrollo local inclusivo desde la prospectiva estratégica en Colombia, la investigación se desarrolló en los Montes de María; subregión compartida entre dos departamentos colombianos. Fue necesario considerar el capital social y relacional, el potencial endógeno, el ordenamiento territorial como herramienta y la priorización de apuestas productivas. Metodológicamente está orientado bajo el paradigma cualitativo, desde la técnica de grupos nominales, matrices de evaluación y selección, donde, la recolección de información fue obtenida mediante entrevistas a informantes clave. La región es un territorio natural, con un gran potencial endógeno, que

Recibido: 23-02-21

Aceptado: 15-06-21

* Economista - CECAR, Magister en Economía - Universidad de Manizales, Magister en Educación Universidad de Sucre, Magister en Negocios Internacionales e Integración - Universidad Tecnológica de Bolívar. Doctorante de Economía en la Universidad del Norte Colombia. Investigadora Asociada categorizada por Min ciencias. Docente investigadora en la Corporación Universitaria del Caribe - CECAR; del grupo de investigación ESAC; claudia.perezpe@cecar.edu.co. ORCID ID. http://orcid.org/0000-0002-3799-9642

* Doctora en Gestión de la innovación Universidad Dr. Rafael Belloso Chacín Venezuela. Investigadora Senior categorizada por Min ciencias. Docente- investigadora del grupo: GORAS y ECOSOL. Universidad Católica Luis Amigó. Medellín - Colombia. Email yamaru.chirinos@amigo.edu.co - urumay78@gmail.com. ORCID ID. http://orcid.org/0000-0003-0471-9859

*** Ingeniero Agrícola - Universidad Autónoma Chapingo, México, Magister en Ciencias en Estrategias para el Desarrollo Agrícola Regional - Colegio de Postgraduados, Puebla, Doctor en Geografía - Universidad Nacional Autónoma Nacional de México. Docente investigador en el Centro Regional Universitario del Noroeste, Universidad Autónoma de Chapingo; gramirezg@taurus.chapingo.mx ORCID ID: https://orcid. org/0000-0002-1711-5942

.*... Doctora en Planificación y Gestión del Desarrollo Regional, Universidad del Zulia Venezuela. Investigadora Asociada categorizada por Min ciencias. Docente Investigadora de la Universidad del Sinú-Elías Bechara Zainum. Montería-Colombia. Correos electrónicos: nataliaberbera@unisinu.edu.co; nataliyabarbera@gmail. com. ORCID ID: http://orcid.org/0000-0002-4566-5052 
depende de actividades agrícolas, ganadería extensiva, actividades manufactureras; la dificultad que se presenta es que la posesión de la tierra está en manos de pocos. Se concluye que, para formular una política pública en la subregión, se requiera implementar por parte del gobierno un plan estratégico de intervención social, que permita la transformación del territorio de forma equitativa, y con impacto social, el cual debe ser viable económicamente, deseable socialmente, soportable ecológicamente, coordinada políticamente y operativamente debe contemplar la construcción, es decir el conocimiento desde la visión de la comunidad; la deconstrucción, a partir de la formulación de proyectos productivos, adecuados a la priorización de necesidades y requerimientos comunales.

Palabras clave: Política pública; desarrollo local; inclusión; ordenamiento territorial.

\title{
Public policy for inclusive local development from strategic foresight in Colombia
}

\begin{abstract}
The article aims to determine elements for the formulation of a public policy of inclusive local development from a strategic perspective in Colombia, the research was developed in the Montes de María; subregion shared between two Colombian departments. It was necessary to consider social and relational capital, endogenous potential, land use planning as a tool, and prioritization of productive bets. Methodologically it is oriented under the qualitative paradigm, from the technique of nominal groups, evaluation and selection matrices, where the collection of information was obtained through interviews with key informants. The region is a natural territory, with great endogenous potential, which depends on agricultural activities, extensive cattle ranching, manufacturing activities; The difficulty that arises is that the possession of the land is in the hands of a few. It is concluded that, to formulate a public policy in the subregion, it is necessary to implement by the government a strategic plan of social intervention, which allows the transformation of the territory in an equitable way, and with social impact, which must be economically viable, desirable socially, ecologically bearable, politically and operationally coordinated, it must contemplate construction, that is, knowledge from the community's vision; deconstruction, based on the formulation of productive projects, appropriate to the prioritization of community needs and requirements
\end{abstract}

Keywords: Public policy; local development; inclusion; territorial planning. 
Pérez Peralta, Claudia Milena; Chirinos Araque, Yamarú del Valle; Sierra Vergara, Leonardo Javier; Barbera Alvarado, Nataliya

Política de desarrollo local desde la prospectiva estratégica en Colombia

\section{Introducción}

Colombia es un país claramente dividido en regiones totalmente disimiles, donde existen insalvables brechas, entre las zonas centrales-privilegiadas y las periféricas-desfavorecidas, tanto a nivel social, económico, e inclusive en la influencia política de toma de decisiones (Salguero: 2004,12). Una de las regiones de Colombia es el Caribe, el cual, constituye un territorio periférico en materia no solo de crecimiento económico, sino de desarrollo social y humano. A su vez el Caribe, está divido en ocho departamentos, entre los que se encuentran, Sucre y Bolívar, los cuales, comparten la subregión Montes de María1.

Esta región natural tiene una extensión total de $6.466 \mathrm{Km}^{2}$, dividida en zona de montaña, zona plana troncal río Magdalena y zona Pie de Monte Occidental. Según el Programa de las Naciones Unidas para el Desarrollo [PNUD] (2003:15), en el documento PROMONTES, el territorio tiene importantes recursos hídricos, representados por aguas oceánicas, aguas de escurrimiento y de infiltración y aguas lénticas; sin embargo, existe vulnerabilidad de escasez de agua en año seco, cuenta con una riqueza forestal importante, y suelos de diversas clases, así como, la presencia de hidrocarburos y recursos mineros en el subsuelo ${ }^{2}$ (ésta es zona de exploración, y tiene redes de transporte de crudo); sin embargo, presenta problemas de erosión, que pueden implicar riesgos a futuro.

Los cultivos tradicionales de la región son yuca, ñame, plátano, maíz, arroz, ajonjolí, aguacate; sin embargo, también se presenta otro tipo de cultivos, que incluso en algunos municipios han entrado a reemplazar los tradicionales, vale decir, que los productos tradicionales, son explotados desde pequeñas unidades familiares que realizan cultivos de pan coger, hasta unidades económicas campesinas que generan pequeños excedentes de explotación. En el caso de los cultivos comerciales, e industriales y de exportación las explotaciones en la subregión giran en torno a la producción de cacao, tabaco, palma de aceite, y en menor medida aji y frijol, sin embargo hay pocas experiencias en materia agroindustrial; en lo relacionado con la palma de aceite, si bien en el municipio de María la Baja, existe desde 2007 una planta extractora de aceite de palma, ésta iniciativa, más que impulsadora del desarrollo, se ha convertido según Gómez (2010) en un proyecto excluyente de las comunidades al priorizar el crecimiento económico y la consolidación de capitales privados.

En el caso del tabaco, que es principalmente producido en Ovejas y el Carmen de Bolívar, aunque es una actividad que se considera generadora

1 Son 15 municipios, 7 de Bolívar (Córdoba, El Carmen de Bolívar, El Guamo, María La Baja, San Jacinto, San Juan Nepomuceno y Zambrano) y 8 de Sucre (Chalán, Colosó, Los Palmitos, Morroa, Ovejas, San Antonio de Palmito, San Onofre y Tolúviejo) (Bocchi, 2011, p. 19; De los Ríos, Becerra \& Oyaga, 2012, p. 10).

2 En las veredas Pijiguay, Almagra, El Tesoro y El Ovejero, del municipio de Ovejas hay presencia de hidrocarburos. 
de empleos, tiene varias dificultades importantes a nivel subregional, al menos siete de las tabacaleras más grandes cerraron operaciones desde hace varios años, como es el caso de C.I. Tayrona que cerró en 2006 (Arcieri, 2006) o de Espinosa Hermanos, que cerró en 2007 (Martínez, 2007), debido principalmente a la violencia que se desató en la subregión, no se puede desconocer el problema social y de salud pública que deriva el consumo de tabaco (Mojica \& Paredes, 2005:2), lo que crea barreras en su producción y comercialización.

De acuerdo al panorama antes descrito, es posible pensar que la región es próspera que genera gran cantidad de empleos y mejor calidad de vida, pero realmente no es así puesto que la mayoría de las tierra están en manos de grandes terratenientes, es así como en lo referente a las iniciativas relacionadas con el tabaco, y principalmente, la palma aceitera, la experiencia en los Montes de María, puedan conDiagramarse en economías de enclave, es decir actividades productivas, desligadas del resto de sectores, donde, se evidencia una economía dual; una desarrollada y la otra atrasada; estos enclaves funcionan a partir de la consolidación de actividades netamente extractivas, de gran impacto medio ambiental y que no generan mayores oportunidades de desarrollo local, debido a que son agentes externos los que controlan dichas actividades (Conning \& Robinson, 2009:364).

En lo concerniente a la ganadería, el problema tiene que ver con el modo de producción, que es extensivo, y depende de la propiedad de la tierra, la cual, para el caso de Colombia tiene altos niveles de concentración (El Gini de tierras es de 0,655) (OXFAM, 2013:7). Además de las dificultades relacionadas con las algunas actividades productivas, el equipamiento de la subregión es otro cuello de botella importante, dado que no existen condiciones de infraestructura básicas para que ésta sea considerada territorio equipado.

Teniendo en cuenta los planteamientos anteriores es evidente el grado de desigualdad, exclusión y pobreza en la que se encuentra sumergida la región, lo que trae como consecuencias carencias en materia de salud, educación, servicios públicos domiciliarios, vivienda y vías de comunicación. Es así como, en promedio el $68,0 \%$ de la población en los Montes de María es pobre por NBI, mientras que el 33,2\% están en estado de miseria. Cabe destacar que hay municipios donde las personas pobres superan el $80,0 \%$. Por otro parte es relevante destacar que casi la mitad (el 47,0\%) de los municipios de los Montes de María tienen un Índice de Calidad de Vida-ICV de entre 40 y 55 puntos sobre 100.

Según datos del Ministerio de Salud y Protección Social, aunque la cobertura en salud desde 2011, en los municipios de la subregión fue relativamente alta del $98,4 \%$, es importante anotar que en promedio el $94,0 \%$ de la población afiliada, pertenecen al régimen subsidiado; es decir, carecen de recursos económicos para pertenecer al régimen contributivo. Por otra parte, es importante resaltar que, según el Ministerio de Educación Nacional, la cobertura neta en primaria, secundaria y media para los municipios de esta región fue relativamente baja (el 66,2\% en 2012), siendo el acceso a educación superior prácticamente nulo, 
Pérez Peralta, Claudia Milena; Chirinos Araque, Yamarú del Valle; Sierra Vergara, Leonardo Javier; Barbera Alvarado, Nataliya

Política de desarrollo local desde la prospectiva estratégica en Colombia

donde el porcentaje de graduados en 2013 representó dentro de la población total el $0,4 \%$ y dentro de la población de 15 a 29 años, el 1,6\%.

Una vez descrito el contexto situacional de la región se evidencia un escenario muy desalentador, que impide el desarrollo y crecimiento local en los Montes de María por consiguiente se plantea como propósito determinar elementos para la formulación de una política pública de desarrollo local inclusivo desde la prospectiva estratégica en Colombia, que permita lograr impacto y transformación social para lo cual se requiere la intervención y compromiso de parte del gobierno, por tanto en este estudio se realizó el análisis correspondiente al capital social y relacional, el potencial endógeno, el ordenamiento territorial como herramienta, la priorización de apuestas productivas y la prospectiva estratégica, en cuanto a la metodología la investigación es de carácter cualitativo, desde un enfoque participativo, la cual, se fundamentó principalmente en la premisa del Colectivo Inteligente, como técnica de Investigación Acción Participativa.

\section{Axiología del desarrollo local inclusivo}

Cuando se habla de desarrollo, según el enfoque clásico ortodoxo, se piensa por lo menos desde las ciencias economicistas, en el concepto de crecimiento económico, asociado principalmente, a la mayor provisión de bienes y servicios dentro de un país, los países llamados desarrollados o del primer mundo, serían aquellos, con valores altos en términos absolutos y/o relativos de su producto interno o nacional bruto; por lo que la aspiración de cualquier país en situación de desventaja económica, o subdesarrollado sería la de llegar a ser un gran productor de bienes y servicios (Pike, Rodríguez-Pose \& Tomaney, 2007:1254).

Para lo cual se requiere, más que una aspiración, que los gobiernos revisen las políticas públicas en torno a los objetivos de desarrollo planteado, de manera que estas proporcionen un ambiente jurídico favorable para la implementación de un plan estratégico de desarrollo local, donde se vea reflejada la inversión en emprendimientos como iniciativas de negocios y mayores oportunidades de empleabilidad mediante la instalación y apertura de PYME. Lograr la inclusión, el crecimiento económico, mejoramiento de la calidad de vida de los ciudadanos, menores índices de pobreza, aumento en el servicio de educación de calidad, salud y seguridad alimentaria.

Cuando la visión de desarrollo baja al contexto local o regional, no se puede considerar como un proceso uniforme; éste está determinado por el espacio geográfico, la comunidad humana implicada o incumbente, las condiciones históricas, la identidad cultural formada, así como, el conjunto de actividades económicas y sociales propias de cada región (Benko \& Lipietz, 1994:22). El desarrollo desde lo regional, se concibe como un proceso de cambio estructural localizado, que se asocia a un permanente proceso de progreso, lo que combina las dimensiones de espacialidad y territorialidad, con la dimensión de humanidad, desde lo individual y colectivo (Boisier, 2001:4).

Los procesos de desarrollo, 
implican que la región y el territorio, se construyan y deconstruyan constantemente, evolucionado desde estadios inferiores, a niveles cada vez más altos de especialización. Se considera que uno de los pasos importantes para iniciar procesos de cambios y transformación a nivel local, tiene que ver con el ordenamiento territorial, que es concebido como un proceso consciente, es "...el resultado de acciones humanas con el propósito de adaptar o adecuar el territorio, buscando con ello..." (Nacimba de la Cruz, 2019), "...alcanzar mejores condiciones en la calidad de vida de las poblaciones..." (Hernández, 2010:98). Este ordenamiento se ejerce mediante políticas públicas, las cuales, se formulan bajo dos enfoques: uno orientado hacia el desarrollo territorial que se ha denominado ordenamiento activo, y el otro hacia la planificación física-espacial que se ha denominado ordenamiento pasivo (Massiris, 2008).

El ordenamiento territorial, debe conDiagramarse como una acción social, dirigido por administraciones públicas funcionales; donde, el buen gobierno sea prerrequisito para la legitimación de los procesos, bajo un esquema de descentralización y democratización de las estructuras de poder (Instituto Interamericano de Cooperación para la Agricultura -IICA, 2003:106). Es importante destacar, que el ordenamiento del territorio para efectos de este estudio, se enfoca en tres teorías de desarrollo regional; 1) la Teoría del Uso del Suelo de Von Thunen (1826), 2) la Teoría de la Localización Industrial de Alfred Weber (1909), y 3) la Teoría de Los polos de Desarrollo de Francois Perroux (1955) (citados en Salguero, 2006: 6-14).

1. Teoría del Uso del Suelo: establece que el territorio debe ordenarse de acuerdo con el uso económico de cada una de sus zonas geográficas; siendo el valor de la tierra variable, desde el uso y a la cercanía de cada zona, con el centro-renta de ubicación.

2. Teoría de la Localización Industrial: plantea que la industria debe localizarse estratégicamente, de acuerdo con la cercanía con los centros de abastecimiento $\mathrm{o}$ de insumos y el mercado.

3. Teoría de Los polos de Desarrollo: que argumenta que en los centros poblados, es donde se desarrollan procesos industriales importantes; cada uno de estos, tiene una jerarquización y están interconectados (diagrama 1) 
Pérez Peralta, Claudia Milena; Chirinos Araque, Yamarú del Valle; Sierra Vergara, Leonardo Javier; Barbera Alvarado, Nataliya

Política de desarrollo local desde la prospectiva estratégica en Colombia

\section{Diagrama 1}

\section{Teorías de Desarrollo Regional estructuradas en 3 planos}

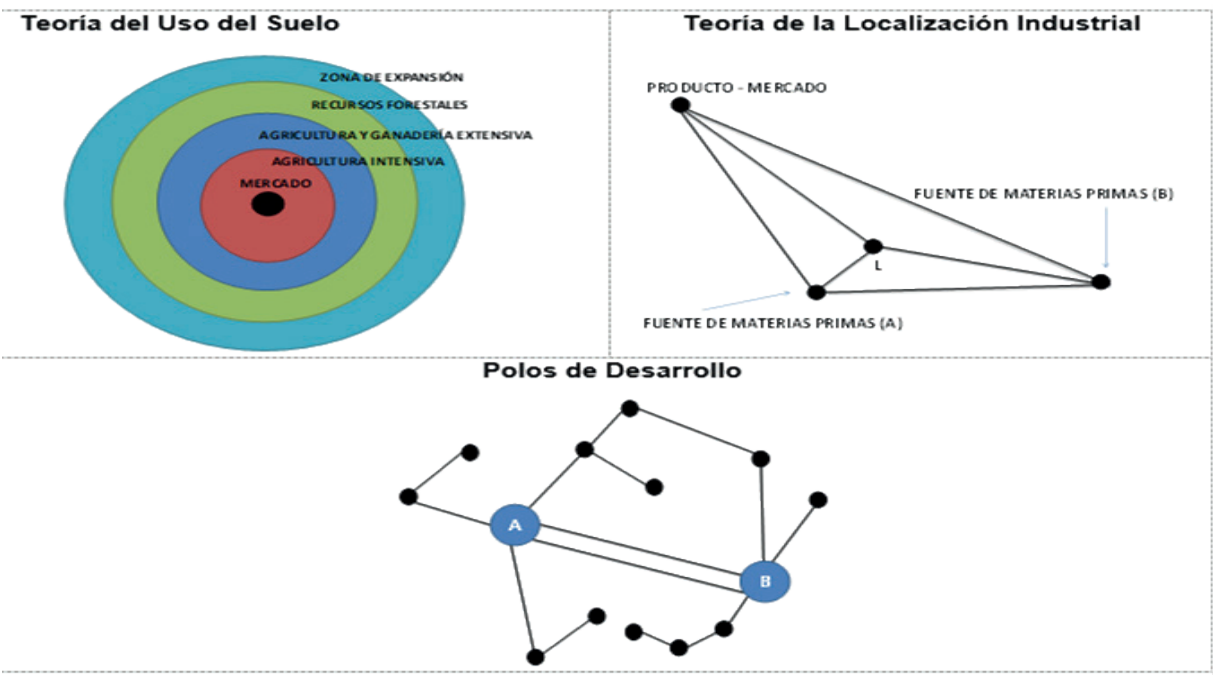

Fuente: Salguero (2006).

El ordenamiento territorial planificado para el desarrollo regional, parte de la construcción social de la visión del territorio, teniendo en cuenta las causas y fuentes de dicho desarrollo. Esta visión surge del análisis objetivo de posibles escenarios futuribles, eligiéndose desde el territorio el escenario apuesta; el sustento de este proceso es el hecho que el futuro no está escrito, ni es uno sólo, sino son varios escenarios posibles, donde las comunidades tienen el poder de escoger y construir su propio escenario (Mojica, 2006: 30-131).

\section{Elementos esenciales para la formulación de una política pública de desarrollo local inclusivo}

Como elementos esenciales para lograr el desarrollo local, destaca la inclusión laboral en los mercados de trabajo locales, sin embargo, en contextos empobrecidos, donde, los mercados de trabajo son fragmentados e ineficientes con escaso desarrollo del tejido empresarial, es necesario generar fuentes de empleo autogestionado; en este orden de ideas, el emprendimiento 
social sostenible, puede ser una estrategia adecuada para el desarrollo local inclusivo.

De acuerdo con Chirinos et al. (2017), el emprendimiento social sostenible es una forma de integración social y económica, alrededor de iniciativas de negocios con innovaciones transformadoras, donde, el colectivo es el principal recurso de producción. Desde una visión creativa, formula y ejecuta un negocio sostenible en el largo plazo, a partir del aprovechamiento de las oportunidades que se presentan en el entorno, donde, el objetivo más que el logro de réditos y rentas individuales, es la generación de empleo, la mejora en la calidad de los servicios sociales, el fomento a prácticas éticas en los mercados (comercio justo) y la sensibilización a nuevos empresarios que quieran hacer una diferencia en la sociedad.

Los emprendimientos sociales, constituyen una innovación social, los cuales, requieren para que sean sostenibles en el tiempo, la construcción de capital social y relacional en los territorios. Es esencial la evidencia de un plan estratégico de desarrollo, implementado por el gobierno, que conduzca a mejora las condiciones de vida de los habitantes, que permita erradicar la pobreza, la exclusión y la violencia en los territorios, donde se evidencie el crecimiento económico local, el desarrollo de capacidades y habilidades técnicas y tecnológicas mediante la de educación de calidad, servicios domiciliaros excelentes, mejores servicios de salud, justicia social y paz.

En síntesis, se puede afirmar que el crecimiento económico puede trascender a un desarrollo local inclusivo, siempre y cuando se generen oportunidades para todas las personas independientemente de su estatus social, género, condiciones físicas, intelectuales, raza (Cordero, 2011:15); de esta manera se incorporan a los ciudadanos vulnerables en las cadenas de valor (Ishikawa \& Strandberg, 2009:7), con el concurso de empresas llamadas ancla que apalanquen el proceso, de acuerdo a las se puede considerar el desarrollo local inclusivo, como un proceso articulado, dinamizado y concertado de todos los actores del territorio, para la transformación local, en pro de la mejora de la calidad de vida de las comunidades, desde su propio potencial y su identidad.

\section{Consideraciones metodológicas de la investigación}

La investigación está orientada bajo el paradigma cualitativo, desde un enfoque participativo, la cual, se fundamentó principalmente en la premisa del Colectivo Inteligente, como técnica de Investigación Acción Participativa; éste consiste en un grupo o grupos de personas plenamente conscientes de su entorno, que mediante su acción, “... amplían la capacidad de los individuos de transformar sus interacciones con su realidad, de manera que provoque en cada persona y en el colectivo, la oportunidad de crear nuevas $y$ diferentes escenarios de vida, en un proceso continuo de evolución y transformación..." (Hennessey, 2005).

El Colectivo Inteligente, desarrolla su acción a partir del trabajo cooperativo, como metodología, para el logro de 
Pérez Peralta, Claudia Milena; Chirinos Araque, Yamarú del Valle; Sierra Vergara, Leonardo Javier; Barbera Alvarado, Nataliya

Política de desarrollo local desde la prospectiva estratégica en Colombia

metas comunes; particularmente, esta metodología consiste en armar grupos de individuos que compartan una realidad, donde cada integrante del grupo ayuda a lograr una meta, que consistiría en determinar los elementos de política para el desarrollo local inclusivo. Ahora bien, como el Colectivo Inteligente debe llegar a consensos para tomar decisiones, la técnica a nivel práctico que se empleó en el proceso para poder llegar a estos acuerdos fue una combinación entre: La Técnica de Grupos Nominales (TGN) y Las Matrices de Evaluación y Selección; estas técnicas combinadas permitieron tomar las decisiones del colectivo acera del potencial endógeno, las necesidades del territorio, la visión prospectiva y las principales estrategias de desarrollo local.

Lo anterior fue implementado en tres ciclos de talleres, dirigidos a diferentes grupos poblacionales, en tres etapas: Construcción, Deconstrucción y Performance; además, existió una fase Preliminar al proceso como tal, que fue de búsqueda y preparación de información y del equipo de trabajo. A nivel práctico, en estos talleres se hizo cartografía social, para determinar y ubicar espacialmente, las potencialidades y limitaciones del territorio, se aplicó el método Potencialidades, Aprovechabilidad, Sustentabilidad y CompetitividadPASC para analizar la forma en que las potencialidades se pueden aprovechar de manera eficiente en el territorio, y un ejercicio de prospectiva estratégica desde el enfoque de Godet (1993; 2007) para construir la visión de territorio.

Además de lo anterior, para informaciones particulares se realizaron entrevistas a informantes clave, que permitieron dar respuesta al objetivo determinar elementos para la formulación de una política pública de desarrollo desde la prospectiva estratégica en Colombia específicamente en la subregión Montes de María.

\section{Elementos esenciales para la formulación de política pública de desarrollo local inclusivo: Resultados para la subregión de los Montes de María Colombia}

A continuación, se presentan cinco elementos esenciales para la formulación de política pública de desarrollo local inclusivo para la subregión de los Montes de María Colombia, como resultado de la investigación; éstos están enfocados de la siguiente manera: a) capital social y relacional como base para la construcción de territorio; b) identificación del potencial endógeno como base de las ventajas comparativas del territorio; c) el ordenamiento territorial, como visión de región construida; d) cadena de valor promisoria basadas en las apuestas productivas con perspectiva de negocios inclusivos, en torno al enfoque del emprendimiento social sostenible, $y, e)$ la prospectiva estratégica como visión de futuro.

\section{a. Capital social como base para la construcción de territorio}

Para Putnam (1995), el capital social es uno de los elementos fundamentales para la formulación de una política pública de desarrollo local inclusivo. Se describe como las características que posee una comunidad: redes, normas, confianza, que facilitan la coordinación, la cooperación para un beneficio mutuo. La confianza es fundamental para la organización coordinada de ideas 
de negocio puesto que representa el intercambio recíproco y solidario entre instituciones y perdonas para la creación de iniciativas de negocios sostenibles y constituye un entramado de relaciones sociales.

En los Montes de María no parece existir una identidad regional construida (PROMONTES, PNUD, 2003:18), la región está por construirse ${ }^{3}$ y para ello, se debe hacer un acercamiento hacia las bases del territorio, que es la construcción de capital social y relacional. Se analizaron cuatro aspectos fundamentales, concebidos desde la perspectiva y sentir de los montemarianos y las montemarianas: proyecto de vida, la felicidad, los miedos y la libertad.

En cuanto al proyecto de vida, el enfoque de los montemarianos y las montemarianas, trasciende de una dimensión individual, a una dimensión comunitaria, donde, el individuo hace parte de un entramado social, que lo determina, el cual se enfoca en la construcción de familia y de comunidad, para el logro de este los habitantes consideran que poseen condiciones internas, relacionadas con las actitudes, aptitudes y conocimientos, pero que carecen de oportunidades externas relacionadas con el apoyo institucional, empresarial, de estamentos públicos, la falta de recursos de índole económico, lo que implica que no existe libertad como enfoque de desarrollo para tener un nivel de ingresos adecuados que le permita vivir dignamente; esta situación es preocupante dado que sería un círculo vicioso de atraso y pobreza; ya que las personas desean mejorar sus condiciones de vida, pero el contexto en el que habitan les impide consolidar su proyecto de vida.

Cabe destacar que el $72,6 \%$ de la población adulta entrevistada consideró que en el municipio donde viven pueden alcanzar o lograr su proyecto de vida, mientras que en el caso de los jóvenes, la mayoría consideró $(98,5 \%)$ que su proyecto de vida es imposible de alcanzar en su municipio; las razones de la respuesta de los jóvenes, se deben al hecho de que la mayoría de los entrevistados quiere ser profesional, y que en los municipios donde viven no existen instituciones de educación superior, con lo cual, deben trasladarse fuera de su municipio.

Los habitantes del territorio son conscientes de que el logro de objetivos empieza por la construcción de estructuras mentales propicias que fomenten la acción; sin embargo, tienen sentimientos de desesperanza con relación a la acción del Estado y de las instituciones públicas para apoyarlos, lo que se ve reflejado en la ausencia de externalidades positivas que propicien ambientes adecuados para el logro de estos objetivos.

Por otra parte, es importante mencionar la concepción de felicidad, miedo y tristeza, aspectos estos manifestados por las personas que conforman la región de estudio, y que son componentes fundamentales a la hora de definir el territorio. Sobre la felicidad, los montemarianos, volvieron

3 Los Montes de María se han visibilizado por distintas circunstancias ajenas a un proceso de regionalización. En un primer momento, fue visible como despensa alimentaria y cuna de la producción tabacalera en el Caribe Colombiano; luego, fue visible por todo el aprendizaje que generó la organización campesina en Colombia; y finalmente, se volvió visible por la violencia que se generó en la zona. 
Pérez Peralta, Claudia Milena; Chirinos Araque, Yamarú del Valle; Sierra Vergara,

Leonardo Javier; Barbera Alvarado, Nataliya

Política de desarrollo local desde la prospectiva estratégica en Colombia

a coincidir en el tema de familia y los afectos; en el caso de las tristezas están ligadas a eventos de muerte, desarraigo y al estar lejos de los seres queridos, en esta comunidad existen fuertes lazos emocionales con la familia; donde, las estructuras familiares como forma de autoafirmación personal constituyen la base de las comunidades. Asimismo, el miedo está ligado al resurgimiento de la violencia, en este sentido, los montemarianos no tienen libertad política por las condiciones de contexto. En tal sentido, es imperante resaltar que el $64,3 \%$ de los adultos entrevistados se siente feliz, y que el $50,0 \%$ se siente realizado como persona.

Teniendo en cuenta lo anterior se indica, que el territorio posee características de modus vivendi, favorables para iniciar procesos de desarrollo; puesto que se evidencia la disposición, deseo y voluntad por parte de los habitantes en sus proyectos de vida, que están enfocados hacia la construcción de familia y de comunidad, para lo cual, los montemarianos tienen actitudes y valores propios de su cultura, que los hacen tener niveles altos de resiliencia y fuertes apegos emocionales hacia la familia, y una autoafirmación personal desde el entorno cercano; además, dentro de la comunidad existen actitudes hacia la cooperación y ayuda, lo que es básico a la hora de construir capital social. Por consiguiente, es importante destacar que, en la región de los Montes de María, la construcción de capital social es indispensable para adelantar procesos de desarrollo local

\section{b. Identificación del potencial endógeno}

Para responder al potencial endógeno de la región Montes de María, se tomó en consideración la información georeferencial y estadística; se desarrollaron talleres directamente con las comunidades y sobre los recursos productivos en el territorio. Se aplicó cartografía social, así como, el método PASC para identificación no solo del potencial endógeno, sino de su uso actual, es decir, su aprovechabilidad, las condiciones que hacen perdurable la explotación del recurso, su sustentabilidad, sus características apetecidas en los mercados indicando competitividad, en el cuadro 1 se muestran los resultados obtenidos acerca de la aplicación del método PASC.

\section{Potencialidad - Aprovechabilidad - Sustentabilidad y} Competitividad

\begin{tabular}{|c|c|c|c|c|}
\hline \multicolumn{5}{|c|}{ PASC región Montes de María } \\
\hline Recurso & Nombre del Recurso & Aprovechabilidad & Sostenibilidad & Competitividad \\
\hline \multirow[t]{2}{*}{$\begin{array}{l}\text { Económico - } \\
\text { Productivo }\end{array}$} & $\begin{array}{l}\text { a. Tierras arables para } \\
\text { la agricultura }\end{array}$ & $\begin{array}{l}\text { Se da la producción de cultivos } \\
\text { tradicionales tales como } \\
\text { yuca, ñame, así mismo, se } \\
\text { da la producción de cultivos } \\
\text { comerciales e industriales: } \\
\text { existen unas cadenas de valor } \\
\text { pobremente desarrolladas }\end{array}$ & $\begin{array}{l}\text { Cooperativas de } \\
\text { productores campesinos }\end{array}$ & $\begin{array}{c}\text { Presencia de } \\
\text { suelos tipo II y III }\end{array}$ \\
\hline & $\begin{array}{l}\text { b. Paisajes variados } \\
\text { (playa y montaña) para } \\
\text { el turismo }\end{array}$ & $\begin{array}{c}\text { Turismo a pequeña escala, dada } \\
\text { la inexistencia de infraestructura } \\
\text { adecuada }\end{array}$ & $\begin{array}{l}\text { Tendencias mundiales del } \\
\text { turismo conservacionista }\end{array}$ & $\begin{array}{l}\text { Riquezas } \\
\text { paisajísticas }\end{array}$ \\
\hline
\end{tabular}




\section{Cont... Cuadro 1}

\begin{tabular}{|c|c|c|c|c|}
\hline \multirow{4}{*}{$\begin{array}{l}\text { Económico - } \\
\text { Productivo }\end{array}$} & $\begin{array}{l}\text { c. Ubicación geográfica } \\
\text { estratégica para los } \\
\text { flujos comerciales }\end{array}$ & $\begin{array}{l}\text { Cercanía a dos ciudades } \\
\text { principales, donde se desarrollan } \\
\text { actividades comerciales e } \\
\text { industriales }\end{array}$ & Existencia de Redes & $\begin{array}{l}\text { Corredor } \\
\text { geográfico de } \\
\text { Comunicación }\end{array}$ \\
\hline & $\begin{array}{l}\text { d. Vocación productiva } \\
\text { (Agricultura y } \\
\text { Ganadería) }\end{array}$ & $\begin{array}{l}\text { Agricultura campesina y } \\
\text { ganadería extensiva }\end{array}$ & $\begin{array}{c}\text { Organizaciones } \\
\text { sociales relativamente } \\
\text { cohesionadas }\end{array}$ & $\begin{array}{l}\text { Conocimientos } \\
\text { ancestrales }\end{array}$ \\
\hline & $\begin{array}{l}\text { e. Presencia de } \\
\text { hidrocarburos y } \\
\text { minerales (carbón) }\end{array}$ & \multirow{2}{*}{$\begin{array}{l}\text { Inicio de actividades de } \\
\text { exploración y explotación }\end{array}$} & \multirow{2}{*}{$\begin{array}{l}\text { Interés por parte de } \\
\text { compañías privadas }\end{array}$} & \multirow{2}{*}{$\begin{array}{l}\text { Acceso a esta } \\
\text { clase de recursos }\end{array}$} \\
\hline & $\begin{array}{l}\text { f. Presencia de fuentes } \\
\text { de energía (gas) }\end{array}$ & & & \\
\hline \multirow{5}{*}{$\begin{array}{c}\text { Medio - } \\
\text { Ambientales }\end{array}$} & $\begin{array}{l}\text { a. Variedad de fauna } \\
\text { (marina) }\end{array}$ & $\begin{array}{c}\text { Pesca artesanal en los } \\
\text { ecosistemas marítimos y de } \\
\text { manglar }\end{array}$ & \multirow{5}{*}{$\begin{array}{c}\text { Interés del estado, } \\
\text { organismos internacionales } \\
\text { y la ciudadanía en la } \\
\text { protección del medio } \\
\text { ambiente, legislación y } \\
\text { normatividades }\end{array}$} & \multirow{5}{*}{$\begin{array}{l}\text { Presencia } \\
\text { de recursos } \\
\text { diferenciados } \\
\text { relativamente } \\
\text { conservados }\end{array}$} \\
\hline & $\begin{array}{l}\text { b. Zonas de reserva } \\
\text { faunística y forestales }\end{array}$ & $\begin{array}{l}\text { Emprendimientos turísticos de } \\
\text { conservación }\end{array}$ & & \\
\hline & $\begin{array}{l}\text { c. Afluentes de agua y } \\
\text { recursos hídricos }\end{array}$ & $\begin{array}{l}\text { Uso del recuso en actividades } \\
\text { agrícolas y pecuarias, existe } \\
\text { vulnerabilidad en año seco }\end{array}$ & & \\
\hline & $\begin{array}{l}\text { d. Suelo con relieve } \\
\text { diverso }\end{array}$ & $\begin{array}{l}\text { Explotación en zona de montaña, } \\
\text { litoral y planicie }\end{array}$ & & \\
\hline & $\begin{array}{l}\text { e. Variedad de flora } \\
\text { (maderables, frutales) } \\
\text { y recursos forestales }\end{array}$ & $\begin{array}{l}\text { Presencia de especies como } \\
\text { la teca, el roble, los cuales, } \\
\text { son explotados de forma } \\
\text { indiscriminada }\end{array}$ & & \\
\hline \multirow{6}{*}{$\begin{array}{l}\text { Social - } \\
\text { Humano }\end{array}$} & a. Movilidad social & $\begin{array}{c}\text { Presencia y acción de } \\
\text { organizaciones y agremiaciones } \\
\text { de distinta naturaleza en el } \\
\text { territorio }\end{array}$ & $\begin{array}{c}\text { Presencia de } \\
\text { organizaciones con } \\
\text { trayectoria }\end{array}$ & \multirow{6}{*}{$\begin{array}{c}\text { Cultura } \\
\text { Montemariana }\end{array}$} \\
\hline & $\begin{array}{l}\text { b. Conocimientos } \\
\text { ancestrales }\end{array}$ & $\begin{array}{l}\text { Saberes sobre las actividades } \\
\text { agrícolas, pecuarias, pesca, etc. }\end{array}$ & $\begin{array}{c}\text { Grupo poblacional } \\
\text { de mayor edad y } \\
\text { conservación de la } \\
\text { oralidad en la transmisión } \\
\text { del conocimiento }\end{array}$ & \\
\hline & c. Capital social & $\begin{array}{l}\text { Confianza entre vecinos y } \\
\text { conocidos }\end{array}$ & $\begin{array}{l}\text { Familiaridad y amistad en } \\
\text { las comunidades }\end{array}$ & \\
\hline & $\begin{array}{l}\text { d. Empatía social y } \\
\text { calidez humana }\end{array}$ & $\begin{array}{l}\text { Amabilidad y hospitalidad como } \\
\text { valores sociales aceptados }\end{array}$ & $\begin{array}{l}\text { Presencia de valores } \\
\text { socialmente aceptados }\end{array}$ & \\
\hline & $\begin{array}{l}\text { e. Riqueza cultural } \\
\text { (folclor, danzas, } \\
\text { expresiones artísticas, } \\
\text { gastronomía, } \\
\text { festividades, etc.) }\end{array}$ & $\begin{array}{l}\text { Existencia de distintas } \\
\text { expresiones culturales }\end{array}$ & $\begin{array}{l}\text { Aceptación de distintas } \\
\text { manifestaciones entre los } \\
\text { grupos eterios jóvenes }\end{array}$ & \\
\hline & $\begin{array}{l}\text { f. Creencias religiosas } \\
\text { judeo-cristianas (Fe } \\
\text { en Dios) }\end{array}$ & Presencia de grupos religiosos & $\begin{array}{l}\text { Iglesias, templos y centros } \\
\text { de culto }\end{array}$ & \\
\hline \multirow{3}{*}{$\begin{array}{l}\text { Político - } \\
\text { Institucional }\end{array}$} & $\begin{array}{l}\text { a. Liderazgo social y } \\
\text { comunitario }\end{array}$ & Presencia de líderes y lideresas & Formación de líderes & \multirow{3}{*}{$\begin{array}{l}\text { Conciencia e } \\
\text { interés por la } \\
\text { comunidad }\end{array}$} \\
\hline & $\begin{array}{l}\text { b. Interés por el bien } \\
\text { común }\end{array}$ & $\begin{array}{l}\text { Proyectos de vida en } \\
\text { consonancia con el bien común }\end{array}$ & $\begin{array}{l}\text { Interés permanente } \\
\text { y generalizado por la } \\
\text { comunidad }\end{array}$ & \\
\hline & c. Seguridad pública & $\begin{array}{l}\text { Disminución del número de } \\
\text { hechos violentos }\end{array}$ & $\begin{array}{l}\text { Estrategias del gobierno } \\
\text { y la fuerza pública por } \\
\text { reestablecer el orden }\end{array}$ & \\
\hline
\end{tabular}

Nota: Modificación y taxonomía de los Autores

Fuente: elaboración propia (2019) a partir de talleres comunitarios. 
Pérez Peralta, Claudia Milena; Chirinos Araque, Yamarú del Valle; Sierra Vergara, Leonardo Javier; Barbera Alvarado, Nataliya

Política de desarrollo local desde la prospectiva estratégica en Colombia

Una vez presentado el potencial endógeno, se procedió a determinar las variables claves para el desarrollo, lo que permite priorizar los recursos productivos, posteriormente, se consideraron los diferentes recursos sobre los que se tiene que tomar la decisión y se examinaron como contribuyen éstos a cada una de las variables. En el subsistema económicoproductivo, existe un sector agrícola campesino de pan coger y la ganadería extensiva, en un esquema de tenencia de la tierra concentrado, donde, no se hace transformación agroindustrial, aunado al encarecimiento de la provisión de bienes en los mercados locales, dado el mal estado de las vías, y la escasez por las afectaciones climáticas, debido a la falta de infraestructura hídrica y de servicios.

Luego de realizar el análisis del contexto antes planteado se determina que para la comunidad Montemariana prevalece el recurso humano, por encima de otros recursos como potencializadores del desarrollo en su región; así mismo se percibe que los recursos económico- productivos y medioambientales, también juegan un papel fundamental para el desarrollo del territorio; en este orden de ideas, la comunidad está consciente de su impacto como tejido social en su propio desarrollo. Asimismo, se analizaron los riesgos y limitaciones presentes en el territorio, que pueden impedir la aprovechabilidad de estos recursos, en aras de mayor competitividad.

\section{c. Ordenamiento e infraestructura del territorio}

El ordenamiento del territorio, se debe hacer desde dos niveles: macrocontexto y microcontexto; desde el nivel micro contextual para el caso de las unidades de explotación agropecuaria; éstas, serán ordenadas bajo el "Modelo de la Finca Montemariana" (diagrama 2), el cual, es una propuesta de desarrollo sostenible que ha promovido la Fundación Red Desarrollo y Paz de los Montes de María - FRDPMM, y se basa en un sistema productivo agroforestal familiar (Aguilera, 2013:26), que garantiza al campesino, dentro de su propia parcela, no solo los bienes básicos para su alimentación, sino también productos con fines netamente comerciales y de transformación. 


\section{Diagrama 2 \\ Finca Montemariana}

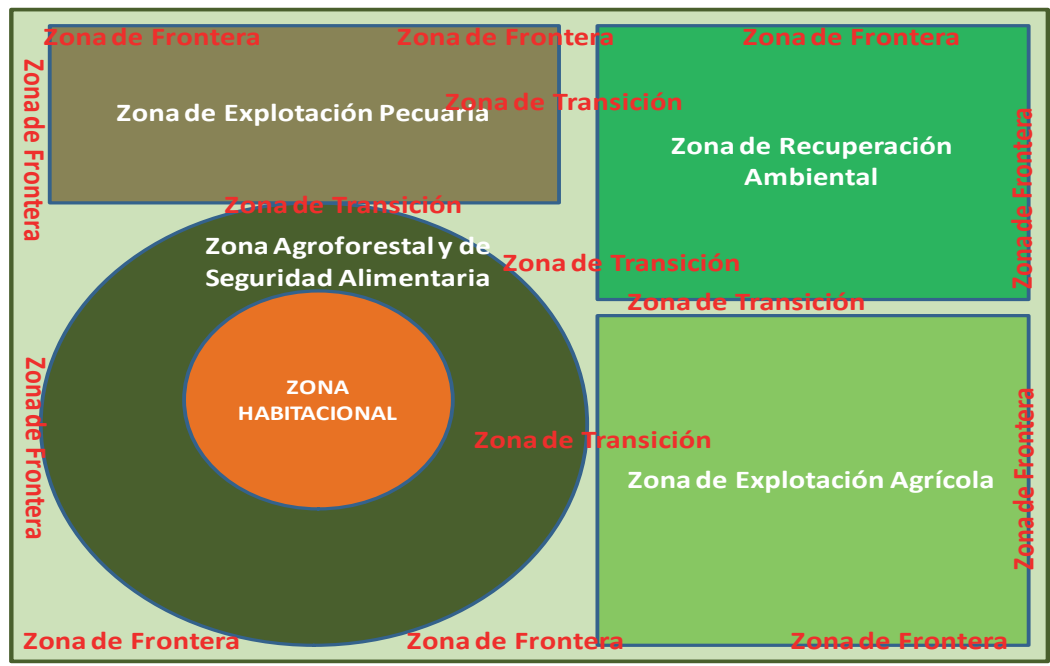

Fuente: elaboración propia (2019) con base en la FRDPMM (2010)

Asimismo, se indica que la zona urbana de los municipios que conforman la subregión, serían centros de acopio de productos, procesos de transformación inicial y comercialización de bienes a pequeña escala; siendo el sector rural, la zona agrícola, pecuaria y forestal (Diagrama 3).

\section{Diagrama 3 Ordenamiento de Montes de María}

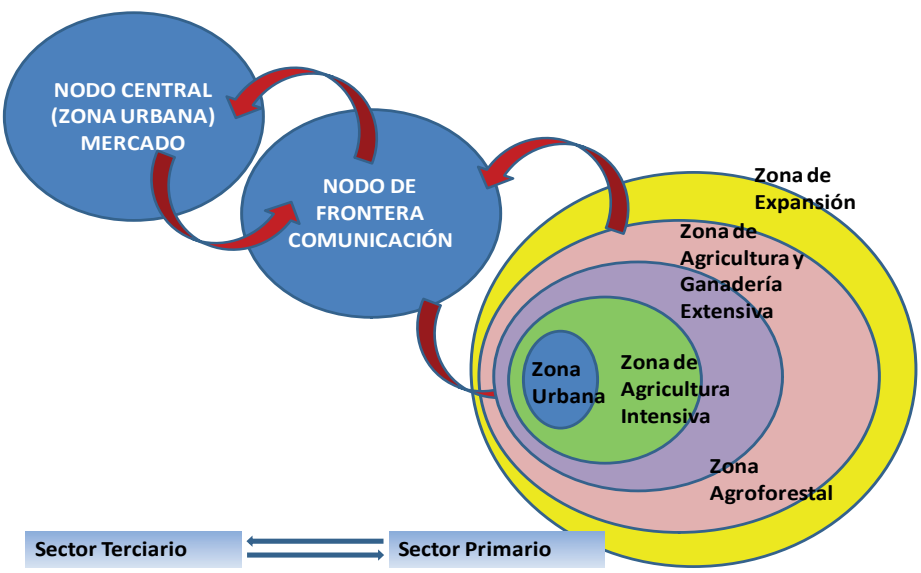

Fuente: elaboración propia (2019) con base en Salguero (2006). 
Pérez Peralta, Claudia Milena; Chirinos Araque, Yamarú del Valle; Sierra Vergara, Leonardo Javier; Barbera Alvarado, Nataliya

Política de desarrollo local desde la prospectiva estratégica en Colombia

A nivel macrocontextual, la región de los Montes de María se podría organizar desde cuatro nodos centrales de diferente tamaño e influencia: la ciudad de Cartagena, la ciudad de Sincelejo y las zonas urbanas de los municipios del Carmen de Bolívar y San Onofre. La ciudad de Cartagena, así como Sincelejo, serían los nodos centrales de mayor tamaño, que agruparían actividades de transformación y comercialización a gran escala de bienes; el Carmen de Bolívar por su parte, sería una unidad zonal de frontera y comunicación, por donde se generaría el transflujo de input y output internodales; otro nodo menor, también de frontera, sería el municipio de San Onofre que conectaría a Toluviejo y María La Baja; configurándose todo en un cúmulo de relaciones trianguladas en el espacio (Diagrama 4).

\section{Diagrama 4}

Influencia de los Nodos Subregionales en el Desarrollo

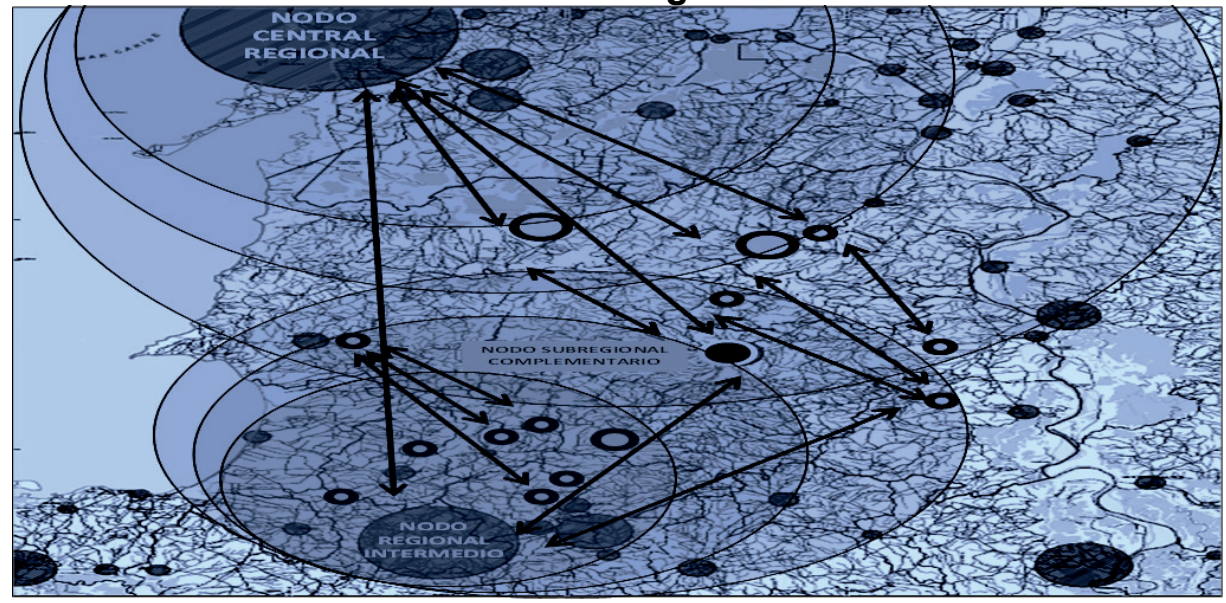

Fuente: elaboración propia (2019) con base en Salguero (2006).

Desde este enfoque se explica que los procesos de desarrollo serían a partir del transflujo de recursos intranodales y los spillover internodales, teniendo en cuenta, que la región de los Montes de María es un territorio con vocación agropecuaria, además, un territorio con riqueza paisajística, donde, se puede acceder a dos nodos comerciales e Industriales importantes como son Mamonal en Cartagena, mucho más desarrollado y el Parque Industrial de Sincelejo; con menos desarrollo, la propuesta en materia económica se fundamenta en dos actividades estratégicas: actividades agroindustriales y el turismo.

\section{d. Cadenas de valor promisorias}

La región Montes de María es un 
territorio con vocación agropecuaria, y con una riqueza paisajística; la propuesta de valor en materia económica se enfoca en dos apuestas: La Agroindustria de productos tradicionales y comerciales, y el Turismo de Naturaleza y Cultural. En cuanto a la transformación agroindustrial, existen posibilidades en torno a productos como ñame, yuca, cárnicos, lácteos, entre otros; en la parte de turismo, la subregión tiene atracciones naturales, como cavernas, saltos y espejos de agua dulce, así como, acceso al mar Caribe; mientras que, en lo cultural, se tiene museos etno-arqueológico, muestras musicales autóctonas y artesanías (Aguilera, 2013:55). Para que este escenario apuesta se materialice se necesita que el tejido empresarial evolucione, permitiéndose la posibilidad de conDiagramar un pre-distrito agroindustrial y turístico para toda la región.

Por consiguiente, se conciben tres fases del proceso:

Fase 1: Empresas Emergentes, donde, es fundamental la implementación de emprendimientos sociales sostenibles, desde un modelo de negocios inclusivos con empresas ancla, que articulen recursos económicos foráneos al territorio que apoyen iniciativas económico-productivas de la región, desde la asociatividad y el cooperativismo;

Fase 2: Empresas en Crecimiento, donde, se presenta la consolidación de encadenamientos productivos en el interior de la región; a partir de la transferencia tecnológica y de base;

Fase 3: Empresas Maduras, desde la formación de un pre-distrito agroindustrial y turístico para la región de los Montes de María. Una vez superada esta fase, se continúa la consolidación de un distrito con todas las implicaciones que el mismo tiene.

\section{e. Prospectiva estratégica como visión de futuro}

De acuerdo al contexto anteriormente descrito, en el marco de esta investigación, se realizó un ejercicio de prospectiva estratégica como visión de futuro para la región de los Montes de María, esta se considera importante porque permite obtener un escenario futuro que no sea fruto del simple proceso de imaginación o de prospección mental de los actores, sino un escenario basado en la cuantificación de las opiniones cualitativas y en las probabilidades, es así como desde el conocimiento del territorio, surgen acciones de políticas públicas, las cuales, tienen como norte la visión de región construida, en un territorio ordenado, empoderado y equipado, que organice sus apuestas en materia de empleo y generación de ingreso.

Desde este ejercicio se pudo evidenciar que las variables estratégicas para el desarrollo son eminentemente productivas y de generación de ingresos; donde, el escenario apuesta para la subregión, consiste en una especialización estratégica localizada, los actores impulsores de este "escenario apuesta”, son los gobiernos extranjeros y el gobierno central, dado que según el territorio las administraciones locales, son actores autónomos y desarticulados, que adolecen de gobernanza y gobernabilidad en la subregión. De acuerdo con las descripciones contextuales antes mencionadas y en concordancia con el ejercicio realizado en la investigación, se presenta el cuadro 2 las variables fundamentales y las hipótesis planteadas por los investigadores que permitieron la 
Pérez Peralta, Claudia Milena; Chirinos Araque, Yamarú del Valle; Sierra Vergara, Leonardo Javier; Barbera Alvarado, Nataliya

Politica de desarrollo local desde la prospectiva estratégica en Colombia

construcción de los escenarios posibles para la formulación de una política pública para el desarrollo local inclusivo en la región Montes de María.

\section{Cuadro 2}

\section{Montes de María. Hipótesis Formuladas.}

\begin{tabular}{|c|c|c|c|}
\hline $\mathrm{N}^{\circ}$ & Variable & Nombre & Hipótesis \\
\hline 1 & VAR1 & $\begin{array}{c}\text { Agroindustria } \\
\text { (Cadenas Productivas) }\end{array}$ & $\begin{array}{l}\text { En el año 2025, la Región Montes de María será un } \\
\text { territorio altamente productivo, donde las cadenas de } \\
\text { valor estarán fuertemente consolidadas, alrededor de } \\
\text { productos de tipo Agroindustrial, haciendo que la Región } \\
\text { sea altamente competitiva. }\end{array}$ \\
\hline 2 & VAR2 & Turismo de Naturaleza & $\begin{array}{l}\text { En el año 2025, la Región Montes de María será } \\
\text { un territorio visitado de forma masiva por turistas } \\
\text { procedentes de otras regiones de Colombia, así como de } \\
\text { otros, convirtiéndose en un destino turístico de naturaleza } \\
\text { reconocido. }\end{array}$ \\
\hline 3 & VAR3 & $\begin{array}{l}\text { Territorio Equipado } \\
\text { (Servicios Públicos } \\
\text { Domiciliarios y Sistema } \\
\text { Vial) }\end{array}$ & $\begin{array}{l}\text { En el año 2025, la Región Montes de María será un } \\
\text { territorio equipado, con infraestructura social y económica } \\
\text { adecuada, que generen condiciones de vida óptimas para } \\
\text { sus habitantes. }\end{array}$ \\
\hline 4 & VAR4 & Fuentes de Empleo & $\begin{array}{l}\text { En el año 202, la Región Montes de María será un territorio } \\
\text { con grandes fuentes de empleo para sus habitantes, lo } \\
\text { que implicará bajas tasas de desocupación y subempleo } \\
\text { en el territorio. }\end{array}$ \\
\hline
\end{tabular}

Fuente: elaboración propia (2019).

En el cuadro 2 se evidencian las cuatro variables estratégicas seleccionadas de acuerdo con las manifestaciones de los habitantes de la región para formular los escenarios; cada variable dio origen a una hipótesis y de la combinación de hipótesis nacieron los posibles escenarios, donde se priorizaron los cuatro primeros, de acuerdo con sus probabilidades de ocurrencia evidenciados en la tabla 1.

\section{Tabla 1}

Montes de María. Posibles Escenarios

\begin{tabular}{|r|r|r|r|}
\hline ESCENARIOS & \multicolumn{1}{|c|}{ PROB. } & \multicolumn{1}{|c|}{$\%$} & \multicolumn{1}{c|}{ \% ACUM } \\
\hline $01-16: 0000$ & 0,481 & 48,1 & 48,1 \\
\hline $02-01: 1111$ & 0,186 & 18,6 & 66,7 \\
\hline $03-11: 0101$ & 0,091 & 9,1 & 75,8 \\
\hline $04-07: 1001$ & 0,099 & 9,9 & 85,7 \\
\hline
\end{tabular}

Fuente: Elaboración propia (2019). 
Teniendo en cuenta los resultados anteriores, se pudieron plantear cuatro posibles escenarios, muy probables; descritos a continuación, según la percepción de los investigadores se considera importante mencionar que los tres primeros escenarios, representan más de las tres cuartas partes de las probabilidades de realización, no obstante, esto no es indicio de que sean los escenarios ideales para la formulación de una política publica para el desarrollo inclusivo en la región Montes de María.

Escenario 1 Todo igual: es decir nada cambia No se cumplen ninguna de las hipótesis planteadas (probabilidad del 0,481$)$, lo cual, constituiría el peor escenario, dado que en la región se están haciendo esfuerzos en materia de desarrollo, desde hace años.

Escenario 2 El Cielo: Se cumplen todas las hipótesis $(0,186$ de las probabilidades), lo cual, sería el escenario ideal, sin embargo, hay que tener en cuenta que la probabilidad calculada de este escenario es muy poca en comparación con el primer escenario.

Escenario 3 Al Natural: Se cumplen las hipótesis relacionadas con el desarrollo del turismo de naturaleza como opción de empleo en la región; sin embargo, la probabilidad de este escenario es de apenas 0,091.

Escenario 4 Agro-Industria: Se cumplen las hipótesis relacionadas con el desarrollo de la agroindustria como fuente de empleo para el territorio; sin embargo, la probabilidad de este escenario es de 0,099. Si se tienen en cuenta los resultados, la probabilidad que las condiciones en la región sigan igual son relativamente altas, lo cual, es hecho sumamente preocupante, ya que a pesar de que existen todas las condiciones en el territorio para su desarrollo, parece perpetuarse estructuras de pobreza no solo material, sino a nivel social, donde, las comunidades viven situaciones de desesperanza, en este caso, el cambio de patrones y actitudes es fundamental.

En síntesis, es relevante destacar que se según los resultados obtenidos de acuerdo a las manifestaciones emitidas por los informantes claves que son habitantes de las comunidades que integran la región objeto de estudio, se evidencia que los tres últimos escenarios, constituyen situaciones ideales para el territorio, cuyas probabilidades sumadas en conjunto representan un 0,376, donde, la región se especializaría en actividades o sector productivos para los que tiene potencial; sin embargo, quedan por fuera aspectos tan importantes como el equipamiento que es fundamental para generar externalidades positivas que atraigan la inversión a la zona.

\section{Consideraciones finales}

Una política de desarrollo local inclusivo en los Montes de María, debe ser capaz de ampliar las libertades de los Montemarianos y las Montemarianas para hacer realidad sus proyectos de vida, puesto que no solo se trata de solventar necesidades básicas, sino de conseguir la generación equitativa e incluyente de oportunidades y la ampliación de capacidades del territorio, que permita la generación de empleos la producción de bienes y servicios que provean calidad de vida a sus habitantes.

La formulación de una política pública debe considerar los elementos esenciales propuestos en este estudio, considerando los escenarios posibles, para lograr que la misma satisfaga las necesidades propias y reales de los habitantes, que se vea reflejada en ella el 
Pérez Peralta, Claudia Milena; Chirinos Araque, Yamarú del Valle; Sierra Vergara,

Leonardo Javier; Barbera Alvarado, Nataliya

Politica de desarrollo local desde la prospectiva estratégica en Colombia

sentir de las comunidades, que venga a resolver los problemas sociales latentes, que conduzca a la transformación social de la región, es así como esta política pública debe estar direccionada al desarrollo y crecimiento económico de la región ser participativa y justa; desde la perspectiva social, debe permitir la evolución hacia sociedades más solidarias; así mismo desde la óptica política, representa la consolidación de regímenes democráticos capaces de mantener la libertad y la paz, en un contexto del postconflicto; y, desde la mirada ambiental, simboliza la puesta en marcha de procesos humanos amigables con el medio ambiente.

$$
\text { Atendiendo a estas }
$$
consideraciones, se recomienda que la apuesta para el desarrollo territorial de los Montes de María debe ser viable económicamente, deseable socialmente, soportable ecológicamente y coordinada políticamente, respecto a esto último, la formulación de una política local de desarrollo inclusivo, debe obedecer a un plan estratégico de intervención regional, con objetivos múltiples, de naturaleza transformadora, conservadora o de compromiso, referido a la distribución más o menos coactiva del poder y los recursos sociales.

Es importante destacar que los Montes de María puede convertirse en una región próspera y segura, puesto que posee oportunidades para la comunidad, pero debe centrarse el desarrollo e impulso del territorio en la generación de fuentes de empleo, donde el turismo sea una de las actividades centrales; un lugar, donde sea garantizada la satisfacción de las necesidades básicas de la comunidad, las urgencias de los montemarianos y las montemarianas, lo que implica equipamiento, tal como, la construcción de instituciones y centros de salud, de educación y de recreación en todos los niveles, así como, la habilitación de vías de acceso, tránsito y comunicación, mediante la pavimentación de calles, caminos y carreteras; teniendo como garante la tranquilidad de la comunidad, donde reina la paz, equidad, inclusión y justicia social.

Para lograr operativizar el desarrollo del territorio sería importante la conjugación de emprendimientos sociales sostenibles, con una base social amplia y organizada con enfoque de red, que establezcan relaciones con firmas externas a la región, desde el enfoque del comercio justo, en un proceso concertado a nivel ciudadano, en el marco de una política pública de desarrollo inclusivo y un esquema de gobernanza territorial multinivel, donde, la meta o fin último sea la mejora en la calidad de vida de la población que se vincula en el ejercicio de la actividad productiva, y por ende de su familia.

Finalmente, según la percepción de los investigadores se considera fundamental que para formular una política pública de desarrollo inclusivo en la región de estudio es importante considerara la implementación por parte del gobierno un plan estratégico de intervención, el cual, operativamente debe contemplar: la construcción, es decir el conocimiento desde la visión de la comunidad y del interviniente acerca del contexto; la deconstrucción, a partir de la formulación proyectos de desarrollo productivo, de acuerdo a la priorización de necesidades y requerimientos; y el performance, desde la validación de procesos, con tiempos, estándares e indicadores claramente definidos. 


\section{Referencias bibliográficas}

Aguilera, M. (2013). Montes de María: Una subregión de economía campesina y empresarial. Documentos de Trabajo sobre Economía Regional, (195). https://doi.org/10.32468/dtseru.195

Arcieri, V. (2006). El Carmen de Bolívar muere por crisis del tabaco. El Tiempo, agosto 24. http://www. eltiempo.com/archivo/documento/ MAM-2148226

Benko, G. \& Lipietz, A. (1994). El nuevo debate Regional" (Introducción), Las Regiones que Ganan, Distritos y redes. Los nuevos paradigmas de la geografía económica. Ediciones Alfons El Magnánim.

Bocchi, D. (2011). Análisis del Plan de Consolidación de Montes de María. Una mirada desde el desarrollo, la democracia, los derechos humanos y la cooperación internacional. Plataforma de Organizaciones de Desarrollo Europeas en Colombia PODEC, (6). http://www.indepaz.org. co/wp-content/uploads/2011/05/599 Podec-6.pdf

Boisier, S. (1991). Gestión regional: un enfoque sistémico. Documento 91/9, Serie Ensayos, ILPES, Chile. (32). https://repositorio.cepal.org/ handle/11362/33652

Boisier, S. (2001). Desarrollo (Local): ¿De qué estamos hablando? en Madoery, Oscar y Vázquez Barquero, Antonio (eds.), Transformaciones globales, Instituciones y Políticas de desarrollo local. Editorial Homo Sapiens, Rosario.

Chirinos, Y., Pérez, C., Barrios, M. \& Martínez, Y. (2017). UniversidadCiencia- Innovación y Sociedad: Desde la perspectiva laboral. Editorial Simón Bolívar. https://bit. Iy/3Afzfhx
Conning, J. \& Robinson, J. (2009). "Enclaves and Development: An Empirical Assessment", St Comp Int. Dev (2009). pp. 359-385. https://doi. org/10.1007/s12116-009-9052-1.

Cordero, M. (2011). Desarrollo Inclusivo Basado en Comunidad: una estrategia para la inclusión social de las personas con discapacidad $y$ el desarrollo de las comunidades desde el enfoque de derechos humanos. Costa Rica, (88). https:// www.academia.edu/4513725/592 desarrollo inclusivo basado en comunidad una estrategia para

De Los Ríos, E., Becerra, C. \& Oyaga, F. (2012). Montes de María. Entre la Consolidación del Territorio $y$ el Acaparamiento de Tierras. Agencia española de Cooperación Internacional para el Desarrollo (AECID), Publicaciones ILSA Bogotá.

Fundación Red Desarrollo y Paz de los Montes de María, FRDPMM (2010). Elementos y estrategia para la construcción de un modelo de desarrollo humano integral sostenible y sustentable para los Montes de María. (Memoria Inédita Contrato $\mathrm{N}^{\circ}$ 0617-1/2010).

Godet, M. (2007). Prospectiva Estratégica: problemas y métodos. Cuadernos de LIPSOR, (20). https://archivo.cepal.org/pdfs/ GuiaProspectiva/Godet2007.pdf

Gómez, A. (2013). La alianza productiva y social de María la Baja en Colombia. Un contraste de lo encontrado con los discursos del desarrollo local. Eutopía. Revista De Desarrollo Económico Territorial, (1), 85-99. https://doi.org/10.17141/ eutopia.1.2010.935

Hennessey, G., (2005). Competencias para crear equipos inteligentes. Revista Latinoamericana de 
Pérez Peralta, Claudia Milena; Chirinos Araque, Yamarú del Valle; Sierra Vergara,

Leonardo Javier; Barbera Alvarado, Nataliya

Politica de desarrollo local desde la prospectiva estratégica en Colombia

Comunicación CHASQUI, (092), 7279. https://revistachasqui.org/index. php/chasqui/article/view/1577

Hernández, Y. (2010). El ordenamiento territorial y su construcción social en Colombia: ¿un instrumento para el desarrollo sustentable? Cuadernos de Geografía, Revista Colombiana De Geografía, (19), 97-109. http:// www.scielo.org.co/pdf/rcdg/n19/ $\underline{\mathrm{n} 19 \mathrm{a} 08 . \mathrm{pdf}}$

Instituto Interamericano de Cooperación para la Agricultura, IICA (2003). El Enfoque Territorial del Desarrollo Rural. San José de Costa rica, agosto de 2003. (139). https://bit. ly/360WzkW

Ishikawa, A. \& Strandberg, L. (2009). Negocios Inclusivos: Creando valor para las empresas y para la población de bajos ingresos. Cuadernos de la Cátedra "La Caixa" de Responsabilidad Social de la Empresa y Gobierno Corporativo, $\mathrm{N}^{\circ}$ 5, IESE Business School. Universidad de Navarra. https://media.iese.edu/ research/pdfs/ESTUDIO-122.pdf

Martínez, L. V. (2007). Cerrarian Tabacalera de Ovejas. El Tiempo, http://www.eltiempo.com/archivo/ documento/MAM-2449098

Massiris, Á. (2008). Ordenación del territorio y desarrollo territorial: la construcción de las geografías del futuro. En, María Angelina Salinas Escobar (Coora). El Ordenamiento Territorial.

Experiencias internacionales. México: Secretaría de Medio Ambiente y Recursos Naturales. Instituto Nacional de Ecología. Centro Universitario de Ciencias Sociales y Humanidades de la Universidad de Guadalajara.

Mojica, A. \& Paredes, J. (2005). Características del cultivo del tabaco en Santander. Ensayos sobre Economía Regional - Banco de la
República, 29(35). https://www. banrep.gov.co/es/caracteristicas-delcultivo-del-tabaco-santander

Mojica, F. (2006). Concepto y aplicación de la prospectiva estratégica. Revista Med, 14(1), 122-131. https://www. redalyc.org/pdf/910/91014117.pdf

Nacimba de la Cruz, K. M. (2019). Modelo de desarrollo urbano sostenible para las nuevas zonas de expansión de El Quinche. Tesis - Magister en Diseño Urbano y Territorial. http://repositorio. puce.edu.ec/handle/22000/17009

OXFAM, (2013). Divide and Purchace. How land ownership is being concentrated in Colombia. Oxfam Research Reports. https://www. oxfam.org/es/node/10130

Pike A., Rodríguez-Pose A. and Tomaney J. (2007). What kind of local and regional development and for whom?, Regional Studies 41, 1253-1269. https://doi. org/10.1080/00343400701543355

Programa de las Naciones Unidas para el Desarrollo et. al., PNUD (2003). Programa de Desarrollo y Paz de los Montes de María. Corporación Territorios Universidad De Cartagena. Convenio Col. 01/054 https://www. co.undp.org/content/colombia/es/ home/library/crisis prevention and recovery/programa-de-desarrollo-ypaz-de-los-montes-de-maria.html

Programa de las Naciones Unidas para el Desarrollo, PNUD (2010). Los Montes de María: Análisis de la conflictividad. Área de Paz, Desarrollo y Reconciliación, Junio de 2010. https://info.undp.org/docs/ pdc/Documents/COL/00058220 Analisis $\% 20$ conflcitividad $\% 20$ Montes\%20de\%20Maria\%20PDF. $\underline{\mathrm{pdf}}$

Putnam, R. (1995). Bowling alone: America's declining social capital. 
pp. $492-513$

Revista Venezolana de Gerencia, Año 26 No. 95, 2021

Journal of Democracy, 6(1), 65$78 . \quad$ https://doi.org/10.1353/ jod.1995.0002

Salguero, J. (2004). Globalización, economía y regiones en Colombia. Sociedad Geográfica de Colombia, Academia de Ciencias Geográficas. Bogotá, 2004. (20). https://www. sogeocol.edu.co/documentos/ GLOBALIZACION.pdf

Salguero, J. (2006). Enfoque sobre

algunas teorías referentes al desarrollo regional. Sociedad Geográfica de Colombia, Academia de Ciencias Geográficas, (20). www. sogeocol.edu.co

Vázquez, A., (2007). Desarrollo endógeno. Teorías y políticas de desarrollo territorial. Investigaciones Regionales,

(11), 183-210. http://www.redalyc.org/articulo. oa?id=28901109 